\title{
TIME TRANSFORMATION METHOD FOR PULSE THERMOGRAPHY
}

\author{
Lukáš MUZIKA, Michal ŠVANTNER, Denis MOSKAL \\ University of West Bohemia, Pilsen, Czech Republic, EU, \\ muzika@ntc.zcu.cz, msvante@ntc.zcu.cz, moskal@ntc.zcu.cz
}

https://doi.org/10.37904/metal.2019.731

\begin{abstract}
Pulse thermography is a method for an inspection of near surface defects. The method is based on heating a tested object with an excitation source (e.g. flash lamp, halogen lamp, laser, microwave) by one pulse. A temperature response is than recorded by a thermographic camera. A result of an evaluation are indications of the defects. The indications are however usually not significant enough or not visible at all without an application of a special data processing methods. A complexity of the evaluation thus often limits a usability of the pulse thermography in industrial applications. The results are often in a form of a sequence composed from many images (typically more than 1000), which should be inspected manually by an operator. We propose and describe a technique, which allows to compress the results to a one image only. This makes an evaluation very fast and very usable for an industrial use. This technique is also suitable for an estimation of depths of the inspected defects. Principles of the proposed evaluation method are described and evaluation results of a steel sample inspection are demonstrated in this contribution. Advantages and disadvantages of the technique are discussed.
\end{abstract}

Keywords: Infrared non-destructive testing, thermal tomography, pulse thermography, defect depth estimation

\section{INTRODUCTION}

Infrared nondestructive testing (IRNDT) is a modern inspection technique used in many different fields of industry. It is can be used for detection of surface and near surface defects for example for detection of corrosion, cracks, delamination and much more in various materials. IRNDT is not limited just for defect detection but it is also used in many cases for defect depth or thermal properties estimation. Over the past decades many different types of IRNDT was developed and established as standard techniques for defect detection in particular areas (e.g. lock-in thermography for solar cells testing [1,2]). The most popular technique remains pulse thermography mostly due to a fast inspection time and relatively simplistic analytical modelling. By pulse thermography, the excitation source (e.g. flash lamp, halogen lamp, electric current) excites the object by one pulse and temperature response on this pulse is than recorded by IR camera. Schematic principle of excitation and thermal response recording is in Figure 1.

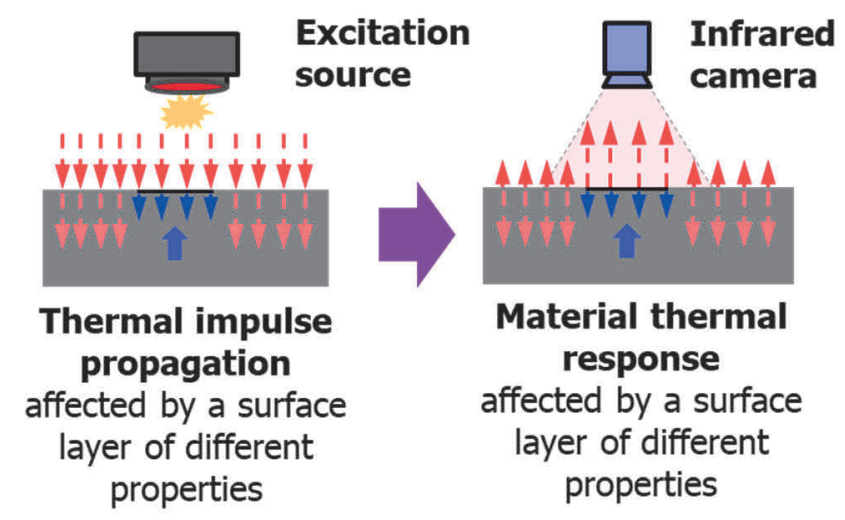

Figure 1 IRNDT system composition 
Just few parameters of measurement can be changed, when the pulse thermography measurement is performed. Those are duration of the recording (number of frames of recorded thermographic sequence) and the speed of acquisition (framerate of IR camera). With some excitation sources (e.g. laser) the pulse length can be also adapted.

In the most common combination, the excitation source for pulse thermography is a flash lamp. The pulse length in this case cannot be changed. It is very appealing for operators that just two parameters can be changed and thus the recording part is very simple. Data recorded by an IR camera are called thermographic sequence. It is basically video but instead of having frames in visible spectrum they are in IR spectrum. A lot of techniques for post-processing to improve defect detectability were developed and has been used on day to day basis. One of the most popular techniques are thermographic signal reconstruction [3], Fourier transform [4] or Wavelet transform [5] based algorithms. Those algorithms provide results in a form of another sequence, which is composed by many frames. Those post-processed sequences are then manually inspected by operator. In practice it means that the operator is searching for one image in the whole post-processed sequence to detect a defect. If defects are in different depth they appear in different sequence image, which makes the evaluation procedure time demanding. Furthermore, it can happen that even though a frame shows clear defect indication it can be easily missed due to tiredness of the operator. From all mentioned above we can conclude that algorithm which would create just one image result, would make an evaluation easier, faster and be beneficial for industrial applications.

Because of that we adapted a technique which gives result in one image. Principles of the technique were introduced in [6,7]. It is based on analysis of an artificial function obtained by multiplying a front-surface temperature evolution by the power function of time. Thus, we call it time power transformation or shortly Pfunction. By this function, the artificial curve is obtained via time transformation from the temperature response on a pulse excitation. This artificial function contains a characteristic point, which moves in dependency of temperature response. If the defect is present, the temperature response and thus characteristic point differs from non-defective zone. The time of occurrence of this characteristic point is saved as a result. The result is in units of time, thereby it is called timegram. This approach was verified mostly on analytical data obtained from some simulation software in previous works and when tested on real data it did not perform that well especially due to noise. In this contribution we introduced our adaptation, which improves results of this method and demonstrated inspection results of real steel sample.

\section{DESCRIPTION OF EXPERIMENT}

Experimental measurement was performed on the steel sample. Dimensions of the sample are $110 \times 70 \times$ $5.15 \mathrm{~mm}$. The sample contains 8 flat bottom holes with diameter from $0.5 \mathrm{~mm}$ to $1 \mathrm{~mm}$ and remaining wall thickness from $0.848 \mathrm{~mm}$ till $3.041 \mathrm{~mm}$. IR camera FLIR SC 7650 was used for thermographic measurement. The framerate was $100 \mathrm{~Hz}$ and 1000 frames were recorded. The sample was excited by $6 \mathrm{~kJ}$ flash lamp. For the post-processing, the P-function was used. There is a parameter which significantly influences the result. This parameter can be from 0 to 0.4 but when it is once found (for the tested material and searched defects) it doesn't change. Nowadays we are searching this parameter experimentally. It means that for discovery of suitable parameter the time transformation is performed for many parameters (in case of steel sample from 0.001 with step 0.001 till 0.1 ). This creates a sequence of timegrams, thus, the evaluation doesn't differ from standard post-processing techniques, when it is performed for one-time measurement. We adapted two changes to get better results. Firstly, we de-noise the data because the noise may vary the minimum and thus the characteristic time. Secondly, we search for minimum instead of searching for inflexion points to get characteristic time. The reason to do so is that several inflexion points can occur on the resulting curve. It is not easy task to determine which inflexion point should be chosen, if the process should be automatic. 


\section{RESULTS}

Table 1 contains diameters, remaining wall thicknesses (RWT), ratios diameter/remaining wall thicknesses (D/RWT) and characteristic times of defects. The parameter diameter/RWT is often used for description of defects [8]. This parameter is used because detectability of defect is not influenced just by the depth but also the size and this ratio takes into consideration both of them. The higher the ratio the better the indication of defect should be. We can see clear dependency between D/RWT and the characteristic time. With increasing ratio D/RWT the characteristic time is decreasing. It is obvious that by defects with same diameter (defects 13 , diameter $1 \mathrm{~mm}$ ) the characteristic time is increasing with defect depth (RWT). It means that for defects with same size and different depth we could determine the depth via calibration curve. Nonetheless when defects differ in size, the depth information cannot be so easily retrieved. We can see it on defects 4,6 and 8 which have the same depth but their characteristic times differ. To obtain depths of defects which differ in size, we would have to know their sizes in advance.

Table 1 Parameters and characteristic times of defects

\begin{tabular}{|c|c|c|c|c|}
\hline Defect & RWT $(\mathrm{mm})$ & Diameter $(\mathrm{mm})$ & D/RWT & Characteristic time $(\mathrm{ms})$ \\
\hline 1 & 0.848 & 1 & 1.179 & 75 \\
\hline 2 & 2.188 & 1 & 0.457 & 104 \\
\hline 3 & 3.418 & 1 & 0.292 & 129 \\
\hline 4 & 2.748 & 0.9 & 0.327 & 119 \\
\hline 5 & 2.518 & 0.8 & 0.317 & 120 \\
\hline 6 & 2.788 & 0.7 & 0.251 & 130 \\
\hline 7 & 3.041 & 0.6 & 0.197 & 134 \\
\hline 8 & 2.711 & 0.5 & 0.184 & 136 \\
\hline
\end{tabular}

Time 0.01-0.16 s

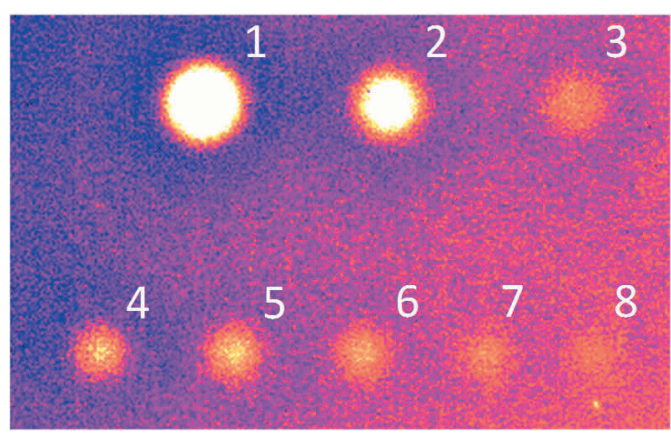

Time 0.09-0.11 s

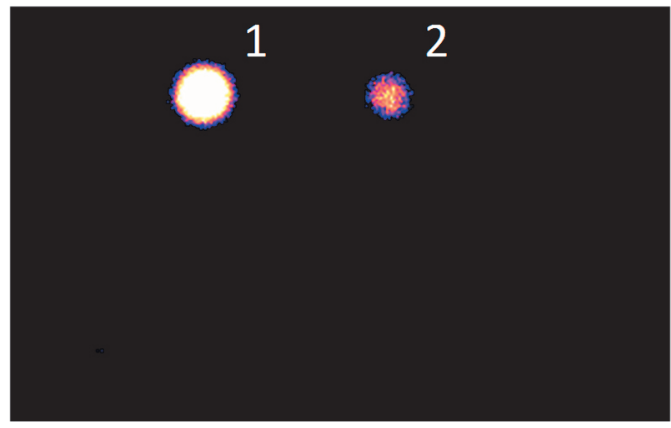

Time 0.08-0.09 s

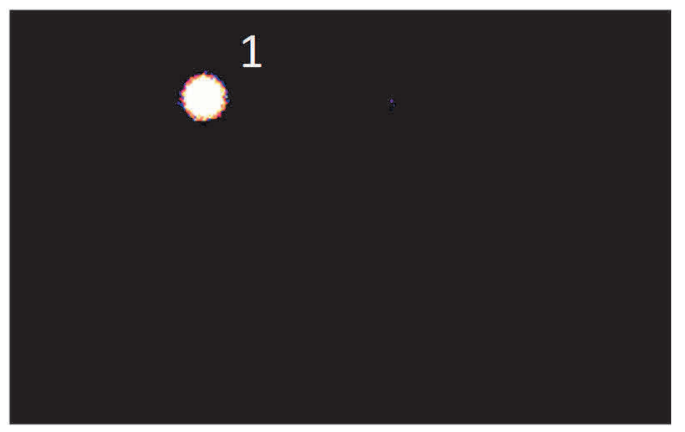

Time $0.11-0.12 \mathrm{~s}$

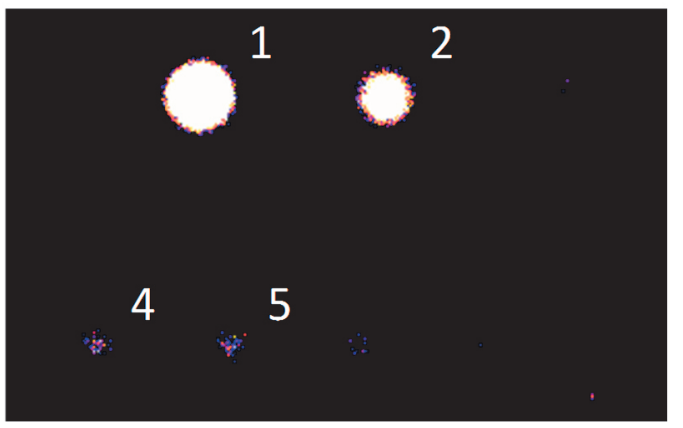

Figure 2 Timegrams with different displayed times 
Results of the inspection are shown in Figure 2. The first timegram shows compressed results, where all defects are indicated in this one picture. Such inspection results are mostly required. Numerical values of the indications on the timegram, i.e. the values of the characteristic times, determine depths and/or D/RWT ratio of the defects. The first timegram with time from 0.01-0.16 $\mathrm{s}$ displays all defects because it displays all characteristic times. This can be visualized by a slicing procedure, which is demonstrated in next timegrams. The slicing is performed via setting specific range of times. Times $t_{1}$ and $t_{2}$ correspond to ratio $r_{1}$ and $r_{2}$ of D/RWT. The lower time means higher ratio. That's why in range $0.08-0.09 \mathrm{~s}$ we can distinguish just the defect 1 , which has the highest D/RWT. With increasing time, we can see defects which have lower D/RWT.

\section{CONCLUSION}

In this contribution the time transformation technique of pulse thermography was used and its abilities were demonstrated on a steel sample. The P-function technique is easy to implement and provides interesting results. The biggest advantage is that the result is in form of just one image. This makes pulse thermography faster and more reliable because operator doesn't have to search an image in the resulting sequence as it is standard for nowadays post-processing techniques. Thus, it saves time of evaluation and minimizes a possibility of missing of an indication on one of many images in a sequence. Furthermore, it is easier to use automatic defect recognition and machine learning, where it is easier to analyze just one frame. The slicing feature is also very interesting for some application. It can be used very well if just defect depths differ and sizes remain the same. In that case a relation between defect depths and characteristic times can be found. If the defect sizes differ, relation between ratio diameter/depth can be found, which can be also interesting for some application.

A characteristic parameter has to be found for an evaluation by the P-function. Nowadays, we are searching for this parameter experimentally. That means that first evaluation is done just for discovering of suitable parameter for given application. It takes approximately same amount of time as standard techniques do (basically instead of sequences of e.g. phases we are investigating timegrams with different parameters). In this case, inspection times are similar as for standard techniques for a one-time measurement. However, the technique is very suited for serial or repeating measurements, because when the parameter of P-function is once found (for the material and defects which are inspected), it doesn't change. P-function thus could be very useful and applicable in wide range of applications.

\section{ACKNOWLEDGEMENTS}

The work has been supported by the Technology Agency of the Czech Republic within the project no. TE01020068 and by the Ministry of Education, Youth and Sports of the Czech Republic within the OP RDI program, CENTEM project, no. CZ.1.05/2.1.00/03.0088, co-funded by the ERDF; and National Sustainability Programme I., CENTEM PLUS project, no. LO1402. and from ERDF "LABIR-PAV / Preapplication research of infrared technologies" (No. CZ.02.1.01/0.0/0.0/18_069/0010018).

\section{REFERENCES}

[1] BREITENSTEIN, O., Illuminated versus dark lock-in thermography investigations of solar cells, Int. J. Nanoparticles. 6 (2013) 81-92. doi:10.1504/IJNP.2013.054983.

[2] MUZIKA, L., ŠVANTNER, M., KUČERA, M., Lock-in and pulsed thermography for solar cell testing, Appl. Opt. 57 (2018) 90-97. doi:https://doi.org/10.1364/AO.57.000D90.

[3] BALAGEAS, D.L., ROCHE, J.-M., LEROY, F.-H., LIU, W.-M., GORBACH, A.M., The thermographic signal reconstruction method: A powerful tool for the enhancement of transient thermographic images, Biocybern. Biomed. Eng. 35 (2015) 1-9., doi: 10.1016/j.bbe.2014.07.002. 
[4] IBARRA-CASTANEDO, C. Quantitative subsurface defect evaluation by Pulsed Phase Thermography, (2005) 188, LAVAL QUEBEC.

[5] KARIMI, M.H., ASEMANI, D., Surface defect detection in tiling Industries using digital image processing methods: Analysis and evaluation, ISA Trans. 53 (2014) 834-844., doi: 10.1016/j.isatra.2013.11.015.

[6] VAVILOV, V.P., NESTERUK, D.A., SHIRYAEV, V. V., IVANOV, A.I., SWIDERSKI, W., Thermal (infrared) tomography: Terminology, principal procedures, and application to nondestructive testing of composite materials, Russ. J. Nondestruct. Test. 46 (2010) 151-161. doi:10.1134/S1061830910030010.

[7] VAVILOV, V.P., KUIMOVA, M.V., Dynamic Thermal Tomography of Composites: A Comparison of Reference and Reference-Free Approaches, J. Nondestruct. Eval. 38 (2019). doi:10.1007/s10921-018-0540-y.

[8] ROTHBART, N., MAIERHOFER, C., GOldAMMER, M., HOHLSTEIN, F., KOCH, J., KRYUKOV, I., MAHLER, G., STOTTER, B., WALLE, G., OSWALD-TRANTA, B., SENGEBUSCH, M., Probability of detection analysis of round robin test results performed by flash thermography, Quant. Infrared Thermogr. J. 6733 (2016) 1-23. doi:10.1080/17686733.2016.1229246. 